\section{RELATIONSHIP BETWEEN THE AGE AND PUBLIC ACCESS DEFIBRILLATION WITH FAVOURABLE NEUROLOGICAL OUTCOMES AFTER OHCA: A PROSPECTIVE, NATIONWIDE, POPULATION-BASED COHORT STUDY}

${ }^{1}$ Kyoko Tsukigase, ${ }^{1,2}$ Hideharu Tanaka*, ${ }^{2}$ Ryo Sagizaka, ${ }^{2}$ Hiroshi Takyu. ${ }^{1}$ Research institute of Disaster management and EMS, Kokushikan University; ${ }^{2}$ Department of EMS system, Graduate school of Kokushikan University, Nagayama, Tama city, Tokyo, Japan

\subsection{6/10.1136/bmjopen-2018-EMS.89}

Aim There is well known on elderly patients decreased good neurological outcome after resuscitation attempt. Aim: The aim of this study was to determine the relationship between the age and post-out-of-hospital cardiac arrest (OHCA) outcomes.

Method Nationwide retrospective cohort study. Settings; A total $1,176,363$ OHCA patients (age $\geqq 15$ years) who were registered in the Japanese Utstein style registry from 2005 to 2014. The primary end point was return of spontaneous circulation (ROSC). The secondary endpoint was 1 month survival with favourable neurological outcomes (cerebral performance categories (CPC) scale; CPC 1-2). In the analysis, risk ratio (RR) and risk difference (RD) with age were calculated and evaluated.

Results Altogether, 296097 cases (25.2\%) were included in the study. According to the RR, we compared the ROSC and age of the patients get PAD at the scene or not. More than 90 years old age wasn't made any differences $(R R=1.55$ (0.92-2.61)). And we also compared the CPC1-2. More than 86 years old age wasn't made any differences. $(R R=2.18$ $(0.92-5.16))$. On the other hand, we compared the RD and got result of the ROSC was more than 72 years old and the CPC1-2 was 71 years old decreased the effectiveness and at the same age of RR made no differences.

Conclusion In Japan, some elderly patients could survive from OHCA with PAD. However, our result suggests that PAD is effective of coming back to the society in patients under 86 years of age.

Conflict of interest None

Funding None

\section{COOLING METHODS OF TARGETED TEMPERATURE MANAGEMENT AND NEUROLOGICAL RECOVERY AFTER OUT-OF-HOSPITAL CARDIAC ARREST}

${ }^{1}$ KH Kim, ${ }^{1}$ SD Shin*, ${ }^{1}$ KJ Song, ${ }^{1}$ YS Ro, ${ }^{2}$ YJ Kim, ${ }^{3} \mathrm{KJ}$ Hong, ${ }^{2} \mathrm{~J}$ Jeong, ${ }^{1} \mathrm{JH}$ Park, ${ }^{1} \mathrm{TH}$ Kim, ${ }^{1}$ SY Kong. ${ }^{1}$ Seoul National University Hospital, S. Korea; ${ }^{2}$ Seoul National University Bundang Hospital, S. Korea; ${ }^{3}$ Seoul National University Boramae Hospital, S. Korea

\subsection{6/10.1136/bmjopen-2018-EMS.90}

Aim The aim of This study is to determine whether the cooling method used in targeted temperature management (TTM) is associated with neurological recovery after out-of-hospital cardiac arrest (OHCA).

Methods Adult OHCA patients transported to emergency departments (EDs) who survived to admission without traumatic etiology and received TTM between January 2008 and December 2016 in Korea were included in the study. Patients without information on neurological status at hospital discharge or without information about the method of targeted temperature management were excluded. Cooling methods were categorized into four groups: external conventional cooling (ECC), such as ice-water spray, fanning and blanket; external device cooling (EDC) using a cooling machine through surface pads; intravascular cooling (IVC) using an intravascular cooling catheter; and intracavitary cooling (ICC) using lavage with ice water. Primary and secondary outcomes were a good cerebral performance scale (CPC) score of 1 or 2 and survival to discharge, respectively. Potential confounders were adjusted in the multivariable logistic regression analysis (reference $=$ ECC).

Results 4,246 eligible patients were included in the final analysis. There was no significant difference in the neurological outcome of a good CPC score between cooling methods compared with ECC; EDC 1.20 (0.95-1.52), IVC $1.43(0.90-2.27)$, and ICC 0.71 (0.46-1.10). The ICC was associated with a poor survival outcome compared to ECC; EDC 0.97 (0.83-1.15), IVC 0.96 (0.78-1.19) and ICC $0.63(0.43-0.85)$. In propensity score matching analysis, there was similar results when comparing outcomes.

Conclusion Cooling methods for TTM did not show any significant difference in neurological recovery.

Conflicts of interest There are no conflicts of interest for all authors in this study.

Funding The study was funded by the Korea Centers for Disease Control and Prevention (CDC). The Korea CDC and National Emergency Management Agency (national fire department) have been collaborating to collect OHCA data. 